\title{
Aberrant methylation of Pax3 gene and neural tube defects in association with exposure to polycyclic aromatic hydrocarbons
}

Shanshan Lin ${ }^{1,2}$, Aiguo Ren ${ }^{1 *}$ (D) Linlin Wang ${ }^{1 *}$, Chloe Santos ${ }^{3}$, Yun Huang ${ }^{1}$, Lei Jin ${ }^{1}$, Zhiwen Li ${ }^{1}$ and Nicholas D. E. Greene ${ }^{3}$

\begin{abstract}
Background: Neural tube defects (NTDs) are common and severe congenital malformations. Pax3 is an essential gene for neural tube closure in mice but it is unknown whether altered expression or methylation of PAX3 contributes to human NTDs. We examined the potential role of hypermethylation of Pax3 in the development of NTDs by analyzing human NTD cases and a mouse model in which NTDs were induced by benzo[a]pyrene (BaP), a widely studied polycyclic aromatic hydrocarbon (PAH).

Methods: We extracted methylation information of PAX3 in neural tissues from array data of ten NTD cases and eight non-malformed controls. A validation study was then performed in a larger independent population comprising 73 NTD cases and 29 controls. Finally, we examined methylation patterns and expression of Pax3 in neural tissues from mouse embryos of dams exposed to $\mathrm{BaP}$ or $\mathrm{BaP}$ and vitamin $\mathrm{E}$.

Results: Seven CPG sites in PAX3 were hypermethylated in NTD fetuses as compared to controls in the array data. In the validation phase, significantly higher methylation levels in the body region of PAX3 were observed in NTD cases than in controls $(P=0.003)$. And mean methylation intensity in the body region of PAX3 in fetal neural tissues was positively correlated with median concentrations of PAH in maternal serum. In the mouse model, BaP-induced NTDs were associated with hypermethylation of specific $\mathrm{CpG}$ sites within both the promoter and body region of Pax3. Supplementation with vitamin E via chow decreased the rate of NTDs, partly recovered the repressed total antioxidant capacity in mouse embryos exposed to BaP, and this was accompanied by the normalization of Pax3 methylation level and gene expression.
\end{abstract}

Conclusion: Hypermethylation of Pax3 may play a role in the development of NTDs; DNA methylation aberration may be caused by exposure to BaP, with possible involvement of oxidative stress.

Keywords: Neural tube defects, Pax3 gene, Methylation, Benzo[a]pyrene, Polycyclic aromatic hydrocarbon

\footnotetext{
*Correspondence: renag@bjmu.edu.cn; linlinwang@bjmu.edu.cn

1 Institute of Reproductive and Child Health, National Health Commission Key

Laboratory of Reproductive Health, and Department of Epidemiology and

Biostatistics, School of Public Health, Peking University Health Centre, Peking

University, Beijing 100191, China

Full list of author information is available at the end of the article
}

C The Author(s). 2019 Open Access This article is distributed under the terms of the Creative Commons Attribution 4.0 International License (http://creativecommons.org/licenses/by/4.0/), which permits unrestricted use, distribution, and reproduction in any medium, provided you give appropriate credit to the original author(s) and the source, provide a link to the Creative Commons license, and indicate if changes were made. The Creative Commons Public Domain Dedication waiver (http://creativecommons.org/publicdomain/zero/1.0/) applies to the data made available in this article, unless otherwise stated. 


\section{Background}

Neural tube defects (NTDs) arise from a failed or disordered closure of the neural tube during embryogenesis. The occurrence of NTDs is around $0.5-2 / 1000$ pregnancies worldwide [1]. Fetuses affected with an NTD are often stillborn (e.g., anencepahlics), and most surviving infants suffer from life-long disabilities. The etiology of NTDs is complex, involving both genetic and non-genetic factors [2]. Over 300 genes have been identified to be involved in the regulation of neural tube closure in mouse NTD mutants [3, 4]; however, only a few of these genes have successfully been validated in human NTDs. In addition to potential additive effects of multiple risk alleles, increasing attention has also focused on the potential for epigenetic alterations to contribute to the occurrence of NTDs by mediating the interplay of fetal genetics and environmental factors [5].

Epigenetic modification can cause changes in gene expression that are not directly related to the DNA sequence itself, of which DNA methylation is one of the best understood epigenetic mechanisms [6]. During early development, a tight regulation of genome-wide erasure of epigenetic footprints with resetting of the methylation signature takes place, making developing fetuses particularly susceptible to epigenetic dysregulation as a consequence of environmental exposure [7, 8]. Abnormal genome-wide methylation during embryogenesis has been linked to developmental abnormalities at birth, including NTDs. Recent studies have demonstrated that global DNA hypomethylation, evaluated using LINE-1 methylation as an indicator in human fetuses, was associated with an increased risk for NTDs [9]. Aberrations of methylation at specific genes are reported to be involved in NTDs, including imprinted genes [10, 11], DNA repair genes [12], planar-cell polarity genes [13, 14], and $H O X$ genes [15].

Pax3 is a paired-homeodomain-containing transcription factor essential for promoting neural crest induction, maintenance, migration, and differentiation [16]. Previous studies in mice have shown that Pax3 function is required for neural tube closure. Several alleles of Pax3 cause NTDs in mice and homozygous Splotch $\left(S p^{2 H}\right)$ embryos develop NTDs with $100 \%$ penetrance [17]. However, the role of PAX3 in human NTDs remains unclear. Recessive and dominant mutations in $P A X 3$ in humans are known to cause Waardenburg syndrome, an autosomal dominant condition that affects neural crest-derived structures and also includes spina bifida as part of its phenotypic spectrum [18]. A 5-bp deletion in exon 5 of the $P A X 3$ gene was reported in a patient with spina bifida [19]. In contrast, the results from a case-control study including 74 infants with spina bifida and 87 non-malformed controls indicated that variants in $P A X 3$ were not strong risk factors for human spina bifida [20]. Therefore, exploring mechanisms other than coding sequence variants, such as methylation modification, in $P A X 3$ may provide novel insight into the etiology of human NTDs.

We hypothesized that aberrant DNA methylation of Pax3 plays a role in the formation of NTDs. To test this hypothesis, we first compared the difference in methylation levels of $\mathrm{CpG}$ sites within PAX3 using genomic methylation array data with DNA from neural tissues of NTD cases and non-malformed controls. The methylation status of the CPG sites that were found to differ was then validated in a larger NTD case-control population. We tested whether there was any correlation between $\mathrm{CpG}$ site methylation levels in fetal neural tissues and maternal serum concentrations of polycyclic aromatic hydrocarbons (PAHs), a class of ubiquitous environmental pollutants that have been shown to be associated with the risk for NTDs in epidemiological studies [21]. Finally, we assessed the methylation level and gene expression of Pax3 in neural tissues from mouse embryos exposed in utero to benzo[a]pyrene (BaP), a widely studied PAH that induces NTDs [22]. In addition, we assayed markers of oxidative stress in fetal mice to further explore the possible mechanisms by which $\mathrm{BaP}$ might affect methylation regulation.

\section{Results}

\section{Methylation of PAX3 gene in genomic microarray}

A detailed description on the genome-wide methylation results obtained from Infinium HumanMethylation450 BeadChip (HM450K), using DNA isolated from neural tissues from ten NTD cases and eight unrelated non-malformed controls can be found elsewhere [23]. In brief, out of 485,199 CpG sites across the entire genome, 23,294 (4.8\%) were differentially methylated between cases and controls. Of the differentially methylated CpG sites, 12,383 (53.2\%) were significantly hypermethylated and 10,911 (46.8\%) were significantly hypomethylated in NTD cases when compared to controls. The characteristics of the NTD cases and controls in phase 1 are presented in Additional file 1: Table S1.

In the PAX3 gene, the focus of this study, a total of 54 CpG sites were extracted from the HM450K array data, of which $47 \mathrm{CpG}$ sites (87.0\%) were found to be hypermethylated. And 7 out of the $47 \mathrm{CpG}$ sites exhibited statistically greater methylation in NTD fetuses than in controls (Additional file 2: Table S2). Analysis of the genomic location of the seven significantly hypermethylated $\mathrm{CpG}$ sites showed that one $\mathrm{CpG}$ was located at TSS1500, one in the 5'UTR, and the remaining five CpGs were within the body of the gene (Fig. 1a).

\section{Validation of PAX3 methylation in an independent cohort of NTD cases and controls}

In order to validate these findings, 73 NTD cases and 29 controls were used to examine the differentially 


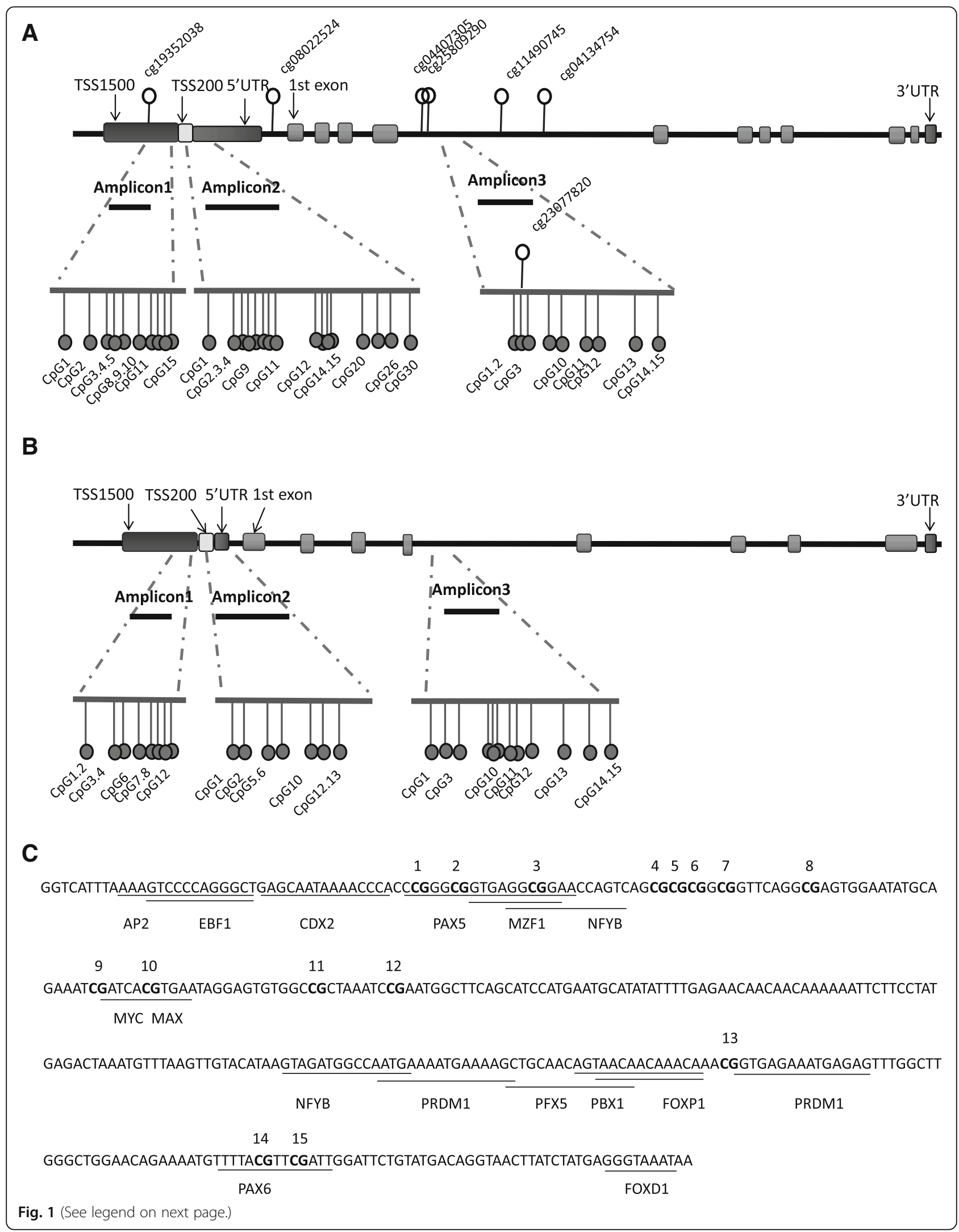


(See figure on previous page.)

Fig. 1 Schematic diagram showing the location of PAX3 region analyzed. a Locations of the CpG sites detected in phase 1 and phase 2 of human methylation analysis. The differentially methylated $\mathrm{CpG}$ units identified by HM450K were indicated as white dots that above the black line (cg19352038, cg08022524, cg04407305, cg25809290, cg23077820, cg11490745, and cg04134754). In phase 2, three DNA amplicons were developed to validate the differentially methylated region detected in phase 1; amplicon 1 covered 10 analytical CpG sites located in the TSS1500 region of PAX3; amplicon 2 covered 16 analytical CpG sites located between TSS200 and 5'UTR region; amplicon 3 covered 9 analytical CpG sites located between the 4th and 5th exon. All the CpG sites detected in replication phase were indicated as black dots under the line. $\mathbf{b}$ Locations of the Pax3 CpG sites detected in mouse methylation analysis. Three DNA amplicons were developed as in human study. Amplicon 1 covered 8 analytical CpG sites located in the TSS1500 region of Pax3; amplicon 2 covered 7 analytical CpG sites which located between TSS200 and 5'UTR region; amplicon 3 covered 12 analytical CpG sites located between the 4th and 5th exon. c Schematic diagram of interest indicating putative transcription factor-binding sties in amplicon 3 of human PAX3 gene in intron 4. CpG sites predicted by JASPAR (predictive value > 8) to bind transcription factors are described under the sequence. CpG site 3 was the same as cg23077820 identified differentially by HM450K in phase 1. All the CPG sites except CpG site 12 were differentially methylated between NTDs and controls by Sequenom EpiTYPER in phase 2

methylated regions of $P A X 3$ identified in phase 1 using Sequenom EpiTYPER. The demographic characteristics of the subjects are described in Table 1. Three DNA amplicons were developed that cover 35 CpGs (Fig. 1a). In the present study, TSS1500, TSS200, 5'UTR, and the 1st exon were defined as the promoter region of $P A X 3$ gene. As shown in Fig. 2a, no significant difference in overall mean methylation levels in the promoter region of $P A X 3$ was observed between NTD cases and controls. However, NTD samples exhibited significantly higher DNA methylation levels $(13.9 \pm 10.1 \%)$ than control samples $(7.8 \pm 4.2 \%)$ in the gene body region $(P=0.003)$ (Fig. 2b). Furthermore, within the gene body, all CpG sites except CpG_12 showed significantly higher levels of methylation in the NTD samples compared to controls (Fig. 2e). After Benjanmini-Hochberg correction, methylation levels in five of the nine CpG sites were still significantly higher in NTD cases.

The relationship between hypermethylation of $P A X 3$ and the risk of NTDs was examined. Methylation levels were categorized according to the median methylation level of the controls. As shown in Table 2, a higher level of methylation in the gene body region was associated with 6.24-fold increased risk for NTDs (95\% CI 1.3029.97).

\section{Correlation between PAH concentrations in maternal serum and PAX3 methylation in fetal neural tissues}

A previous study from our team found that higher $\mathrm{PAH}$ concentrations in maternal serum were associated with an increased risk for NTDs [24]. We therefore conducted a correlation analysis between PAH concentrations in maternal serum and $P A X 3$ methylation levels in fetal neural tissues ( $N=51$ mother-fetus pairs) to further our understanding of the potential relationship of PAH exposure, gene methylation, and NTDs risk. Notably, a significant positive correlation was found between mean methylation levels in the $P A X 3$ gene body and median concentrations of high-molecular-weight PAHs in maternal serum $(r=0.310 . \quad P=0.027)$ (Additional file 3: Table S3).

\section{Disturbed methylation of Pax3 in mouse embryos exposed to BaP}

We further investigated the finding of a correlation between maternal serum PAH concentrations and $P A X 3$ hypermethylation in humans by utilizing a BaP-induced NTD mouse model. Three amplicons developed according to the mouse genome were used (Fig. 1b). The body region of $\mathrm{Pax} 3$ showed a trend toward higher levels of methylation in the BaP-treated group compared with controls (Fig. 3a), but this did not reach statistical significance. Furthermore, in terms of specific CpG sites, two significantly hypermethylated $\mathrm{CpG}$ sites within the promoter and two within the body region of $\operatorname{Pax} 3$ were respectively detected in the $\mathrm{BaP}$-exposed mouse fetuses when compared to the non-exposed fetuses (Fig. 3b-d).

\section{The role of oxidative stress in BaP-related disturbed Pax3 methylation and expression}

Since BaP exposure was hypothesized to favor the generation of reactive oxygen species (ROS) in the embryos, we inferred that oxidative stress may get involved in the aberrant Pax3 methylation induced by BaP. As shown in Fig. 4, compared to the control group, the levels of total antioxidant capacity (TAC) and the activity of superoxide dismutases (SODs) were decreased. Real-time PCR and whole mount in situ hybridization showed that $\mathrm{BaP}$ exposure significantly decreased the transcription of Pax3 ( $P=0.008)$ (Fig. 5). After co-supplemented with vitamin $\mathrm{E}$, a commonly used antioxidant, the repressed levels of TAC and SODs were partly restored (Fig. 4). Coincidentally, the hypermethylation of $\mathrm{Pax} 3$ in specific $\mathrm{CpG}$ sites induced by $\mathrm{BaP}$ treatment was recovered and Pax3 expression was also normalized by vitamin E supplementation (Figs. 3 and 5), which suggested a causal effect of oxidative stress on both $\operatorname{Pax} 3$ methylation and expression.

\section{Discussion}

Pax3 is a key gene, encoding a transcription factor required for neural tube closure [17]. In this study, we evaluated the methylation status of $P A X 3$ in a two-phase 
Table 1 Characteristics of NTD cases and controls in phase 2 for methylation assay

\begin{tabular}{|c|c|c|c|}
\hline Characteristic & Control $(N=29)^{a}$ & Case $(N=73)^{a}$ & $P$ value $^{\mathrm{b}}$ \\
\hline \multicolumn{3}{|l|}{ Maternal age (years) } & 0.703 \\
\hline$<25$ & $14(50.0)$ & $30(41.1)$ & \\
\hline $25-29$ & $6(21.4)$ & $20(27.4)$ & \\
\hline$\geq 30$ & $8(28.6)$ & $23(31.5)$ & \\
\hline \multicolumn{3}{|l|}{$\mathrm{BMI}\left(\mathrm{kg} / \mathrm{m}^{2}\right)$} & 0.595 \\
\hline$<18.5$ & $2(6.9)$ & $6(8.6)$ & \\
\hline $18.5-27.9$ & $22(75.9)$ & $57(81.4)$ & \\
\hline$\geq 28$ & $5(17.2)$ & $7(10.0)$ & \\
\hline \multicolumn{3}{|l|}{ Maternal education } & 0.005 \\
\hline Primary or lower & $4(13.8)$ & $9(12.5)$ & \\
\hline Junior high & $12(41.4)$ & $52(72.2)$ & \\
\hline High school or above & $13(44.8)$ & $11(15.3)$ & \\
\hline \multicolumn{3}{|l|}{ Occupation } & $<0.001$ \\
\hline Farmer & $14(48.3)$ & $61(87.1)$ & \\
\hline Non-farmer & $15(51.7)$ & $9(12.9)$ & \\
\hline \multicolumn{3}{|c|}{ Previous birth defects history } & 0.200 \\
\hline Yes & 0 & $4(5.6)$ & \\
\hline No & $28(100)$ & $67(94.4)$ & \\
\hline \multicolumn{3}{|l|}{ Gravidity } & 0.219 \\
\hline 1 & $17(58.6)$ & $32(45.1)$ & \\
\hline$\geq 2$ & $12(41.4)$ & $39(54.9)$ & \\
\hline \multicolumn{3}{|l|}{ Parity } & 0.060 \\
\hline 1 & $18(72.0)$ & $32(50.0)$ & \\
\hline$\geq 2$ & $7(28.0)$ & $32(50.0)$ & \\
\hline \multicolumn{3}{|c|}{ Periconceptional folic acid supplementation } & $<0.001$ \\
\hline Yes & $4(14.3)$ & $42(60.0)$ & \\
\hline No & $24(85.7)$ & $28(40.0)$ & \\
\hline \multicolumn{3}{|l|}{ Cold or fever } & 0.059 \\
\hline Yes & $5(17.9)$ & $27(37.5)$ & \\
\hline No & $23(82.1)$ & $45(62.5)$ & \\
\hline \multicolumn{3}{|c|}{ Active or passive smoking } & 0.210 \\
\hline Yes & $15(55.6)$ & $29(41.4)$ & \\
\hline No & $12(44.4)$ & $41(58.6)$ & \\
\hline \multicolumn{3}{|l|}{ Drinking } & 0.940 \\
\hline Yes & $17(65.4)$ & $47(66.2)$ & \\
\hline No & $9(34.6)$ & $24(33.8)$ & \\
\hline \multicolumn{3}{|l|}{ Gestational age (weeks) } & 0.023 \\
\hline$<28$ & $14(48.3)$ & $52(72.2)$ & \\
\hline $28-36$ & $7(24.1)$ & $14(19.4)$ & \\
\hline$>36$ & $8(27.6)$ & $6(8.3)$ & \\
\hline \multicolumn{3}{|l|}{ Fetal sex } & 0.129 \\
\hline Male & $17(58.6)$ & $28(41.8)$ & \\
\hline Female & $12(41.4)$ & $39(58.2)$ & \\
\hline
\end{tabular}

${ }^{2}$ Data were presented in number (percentage). Total number may not be equal to the total of cases or controls due to missing or unknown data

${ }^{\mathrm{b}}$ Cases and controls were compared by Pearson's $x^{2}$ test, or Fisher's exact test if any cell expectation was less than 5 design study to examine whether there is a potential role of PAX3 methylation in the development of human NTDs. Differentially hypermethylated CpG sites were found in the promoter and gene body region within PAX3 in the neural tissues of NTD cases. Association analysis showed that a higher methylation level in the gene body region was associated with an elevated risk for NTDs. Moreover, PAH concentrations in maternal serum, which were known to be associated with increased risk of NTDs, were positively correlated to methylation levels at several $\mathrm{CpG}$ sites. In the BaP-induced NTD mouse model, hypermethylation of Pax3 gene and suppressed gene expression were observed in embryos with $\mathrm{BaP}$ treatment, along with reduced TAC level. We previously showed that vitamin E supplementation could decrease the rate of NTDs and alleviate oxidative stress in BaP exposed embryos [22]. Here, we further demonstrated that vitamin $\mathrm{E}$ mitigated the shifts in Pax3 methylation and gene expression.

Studies on the role of the Pax3 in NTDs have mostly focused on gene depletion or loss of function mutants [17]. In mice, mutations in Pax3 give rise to the Splotch $(S p)$ phenotype, which includes exencephaly, spina bifida, and neural crest abnormalities in homozygous mutant embryos $[25,26]$. The human $P A X 3$ gene exhibits high homology with mouse Pax3. Mutations within the human PAX3 gene have been found in Waardenburg syndromes, a condition which is occasionally associated with NTDs [18]. A previous study identified two spina bifida patients who had small interstitial chromosomal deletions involving PAX3 [27]. Exon sequencing of $P A X 3$ in 114 cases with spina bifida also identified two common variants; however, without unaffected individuals, the influence of these variants on the risk of spina bifida could not be determined [28]. To the contrary, the screening results from 74 spina bifida cases and 87 control infants suggested that variant in $P A X 3$ was not a major contributor to the overall burden of NTDs at population level [20]. Recently, evidence from animal studies suggested that aberrant methylation of Pax3 was involved in the development of NTDs induced by hyperglycemia [29, 30]. However, to date, no study investigating the role of $P A X 3$ methylation in the etiology of human NTDs has been reported. In our study, we analyzed the methylation status of $P A X 3$ gene in neural tissues from human fetuses, and our results showed that the methylation level in $P A X 3$ was significantly higher in NTD cases than in non-malformed controls.

Unexpectedly, in our study, compared with promoter region, the body region of $P A X 3$ was more consistently hypermethylated in NTD cases and the differentially methylated region was mainly located in intron 4 . Our results imply that a methylation change in the body 


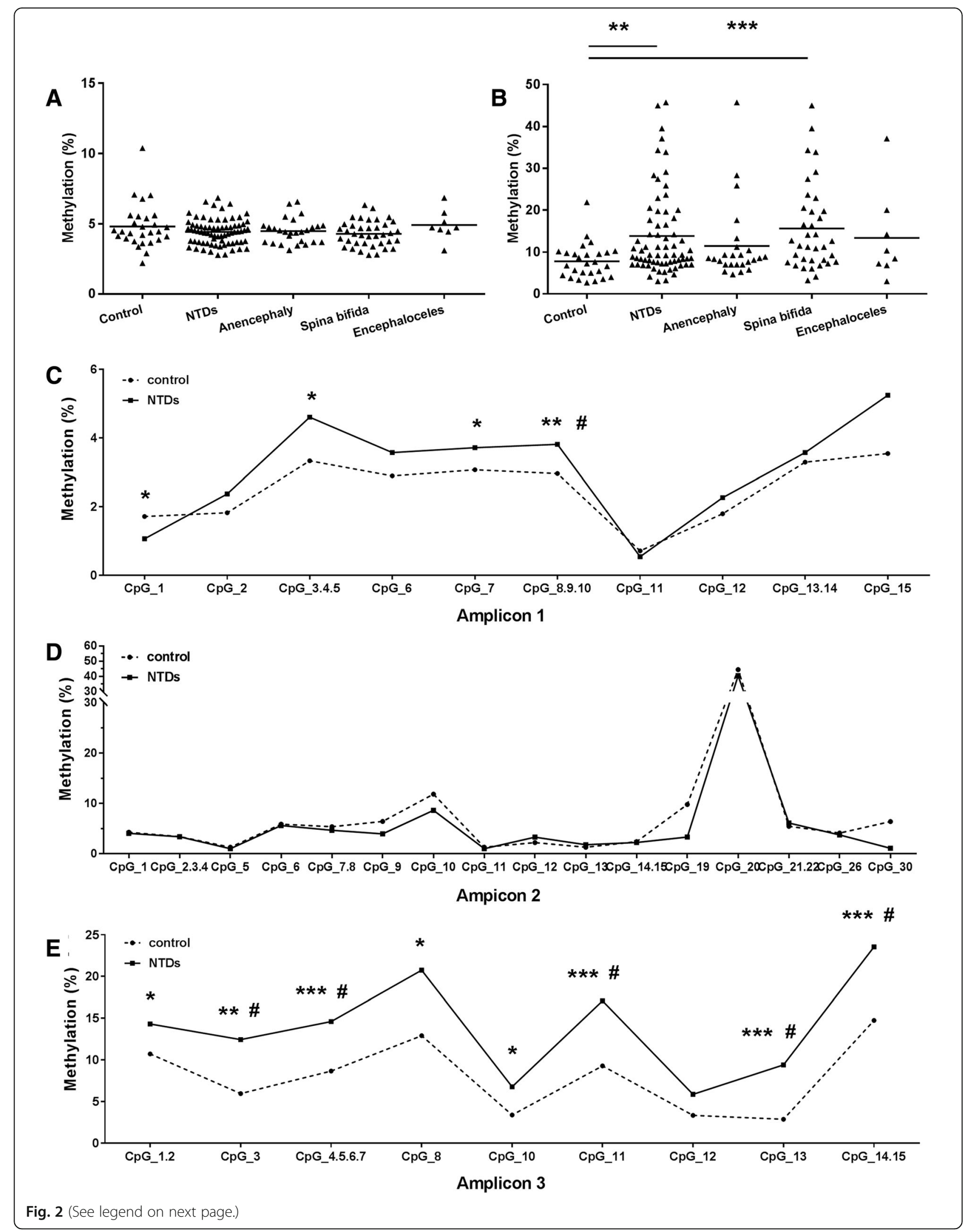


(See figure on previous page.)

Fig. 2 PAX3 methylation pattern assayed by Sequenom EpiTYPER in NTD cases and controls. a Mean methylation level of PAX3 promoter region (amplicon 1 and amplicon 2) between NTD cases and controls. b Mean methylation level of PAX3 body region (amplicon 3) between NTD cases and controls. c-e Methylation levels for each CpG site between NTD cases and controls in promoter (c, d) and body region (e). TSS1500, TSS200, $5^{\prime} \cup R T$, and the 1st exon were defined as the promoter region of PAX3 gene in this study. The significance of differences was calculated using Independent t-test. ${ }^{*} P<0.05,{ }^{*} P<0.01,{ }^{* * *} P<0.001$, compared with control group. ${ }^{\#} P<0.05$, compared with control group after FDR adjustment

region of $P A X 3$ may be an epigenetic component of human NTDs, which is reminiscent with findings for the HOXB7 gene in a myelomeningocele (spina bifida) case-control study [15]. In the latter study, a genome-wide methylation assay found three CpGs in HOXB7 gene body to be hypomethylated in myelomeningocele patients when compared to controls, and these were further verified in a larger population, using the Sequenom EpiTYPER platform. Studies on GRHL3 and SOX18 also reported that altered methylation within the gene body was associated with the risk for NTDs $[14,31]$.

It is generally accepted that DNA methylation changes in the promoter and gene body regions may have differential effects on gene expression. DNA methylation in promoter regions is usually negatively associated with gene expression, whereas in the gene body, the impact is not consistent, with both positive and negative impacts having been reported [32, 33]. In the present study, a negative correlation between gene body DNA methylation and transcription level of Pax3 was observed in mouse embryos, which was in line with the finding in hyperglycemia-induced NTDs [29]. The underlying mechanisms of gene body methylation in regulating gene expression have not been well understood. Recently, increasing evidence demonstrating the role of DNA methylation in alternative splicing regulation has been reported, which is essential for providing tissue-specific features for some genes [34]. DNA methylation is normally more abundant in exons compared to the flanking introns, which is a marker for distinguishing exons from introns. Previous studies proposed that the change of exon methylation levels would affect the recognition of exons while splicing [35]. It is thus reasonable to hypothesize that the methylation status of intron might also be crucial for alternative splicing. Further, cis-acting element has been recognized in intron, which could regulate gene expression [34]. Within intron 4 of $P A X 3$, a number of transcription factors were predicted to bind at several CpG sites examined in the present study, with high predictive values (predictive value $>8$, JASPAR, Fig. 1c), which might be responsible for the observed repressed gene expression. However, more research is necessary to examine this point in detail.

PAX3 hypermethylation by itself is not likely to be a sole cause for NTDs but rather be part of a complex combination of environmental and epigenetic risk factors. Our previous epidemiological studies have

Table 2 Risks of NTDs associated with methylation level of PAX3 gene in fetal neural tissues

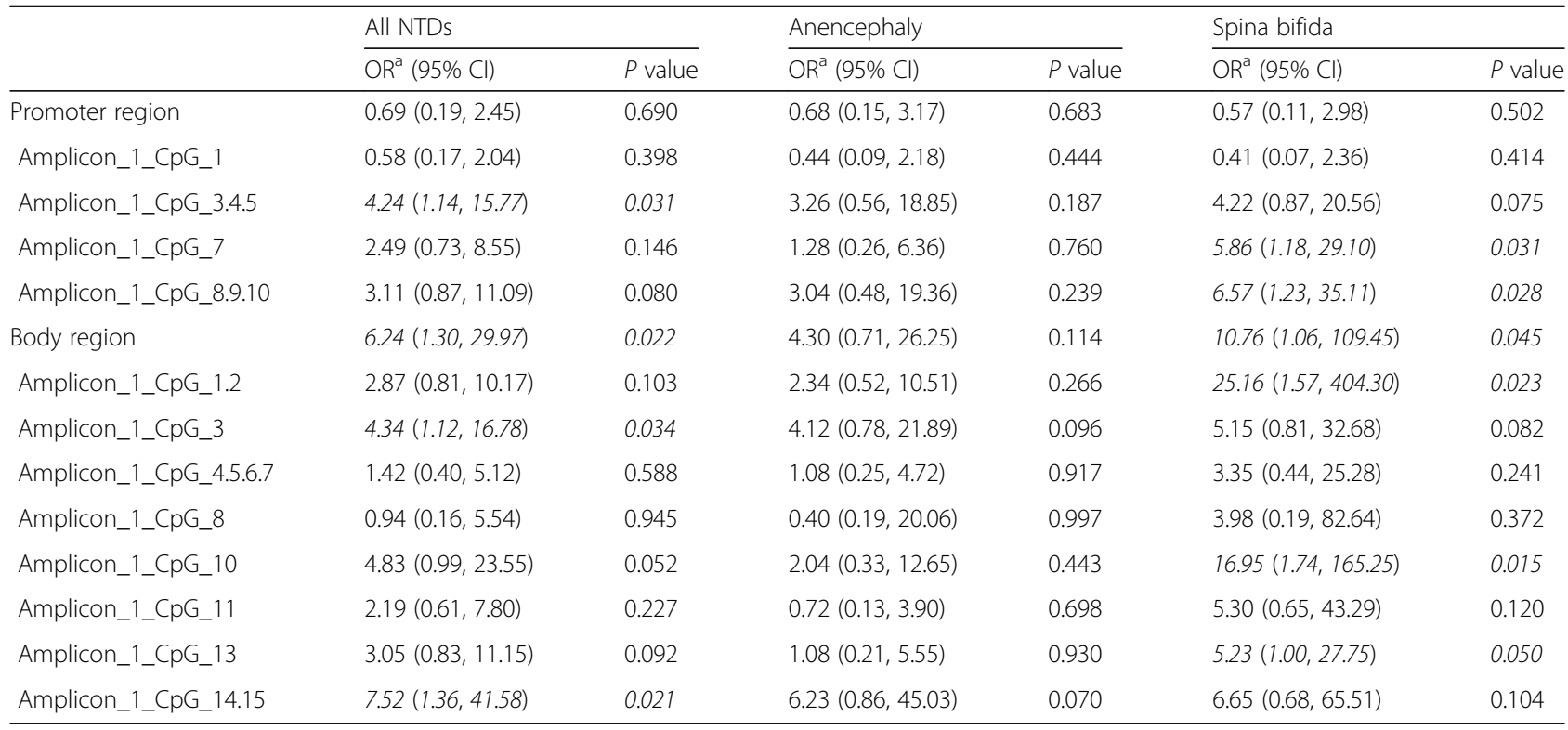

${ }^{\mathrm{a}} \mathrm{OR}$ was adjusted by maternal education, occupation, periconceptional folic acid use and fetal sex, gestational age. ORs were in italic when $P$ value $<0.05$ 

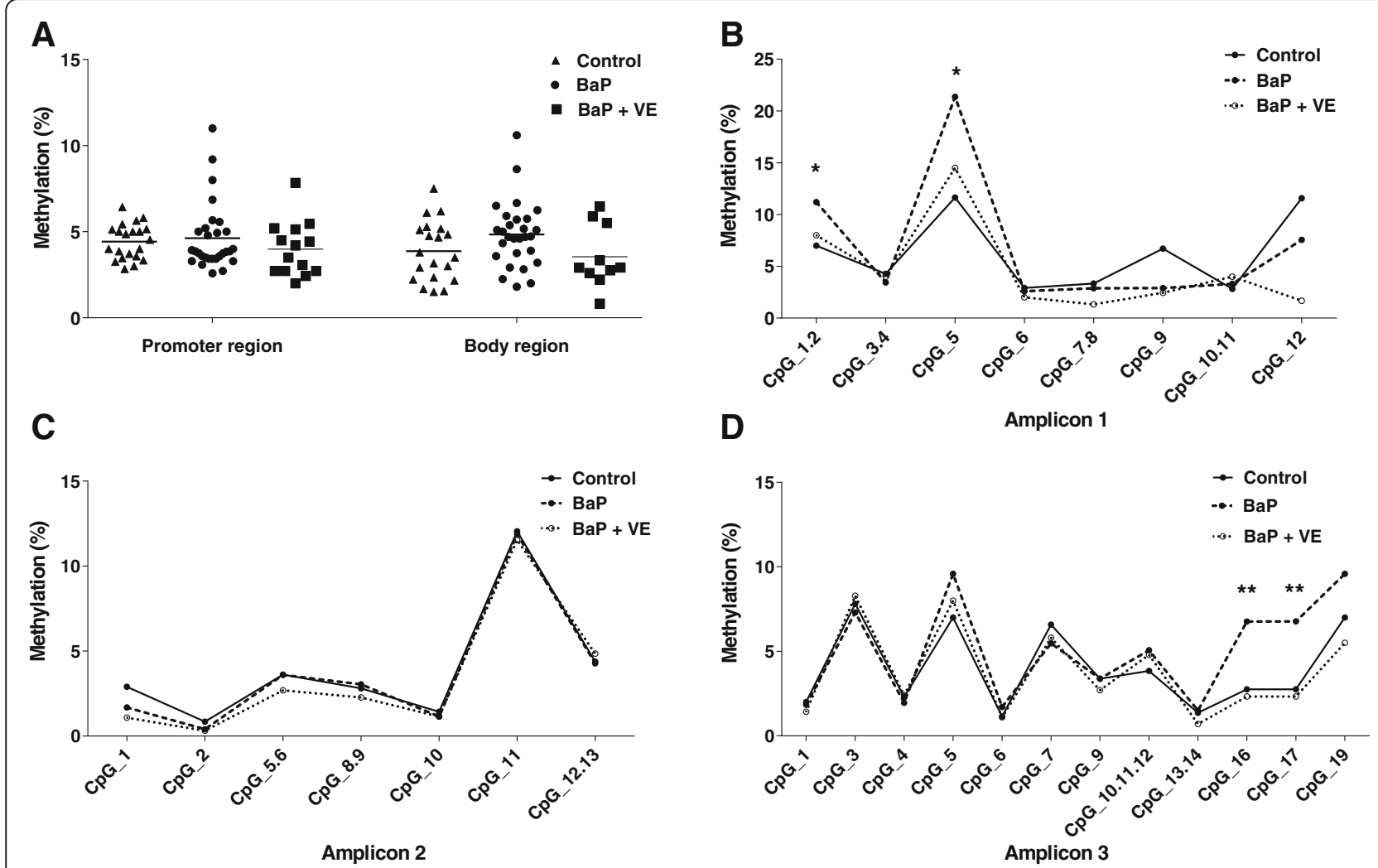

Fig. 3 The effects of BaP and vitamin E on Pax3 methylation in E10.5 mouse embryos. a Mean methylation level of promoter region (amplicon 1 and amplicon 2) and body region (amplicon 3) within Pax3. b-d Methylation level for specific CpG sites in promoter region (b, c) and body region (d) among control group, BaP-treated group and vitamin E co-supplementation group ( $n=24-28)$. TSS1500, TSS200, 5'URT, and the 1st exon were defined as the promoter region of $P$ ax3 gene in this study. The significance of differences was calculated using ANOVA. ${ }^{*} P<0.05,{ }^{* *} P$ $<0.01$, ${ }^{* * *} P<0.001$, compared with control group. VE vitamin $\mathrm{E}$

suggested that maternal exposure to PAHs was associated with an elevated risk of NTDs in the offspring [21, 24]. Cohort studies reported that prenatal PAH exposure was associated with lower global methylation and hypermethylation of interferon $\gamma$ in umbilical cord white blood cells $[36,37]$. Several toxicological studies have also suggested that $\mathrm{BaP}$ exposure could disrupt DNA methylation status $[38,39]$. Importantly, in the present study, we found that differentially methylated $\mathrm{CpG}$ sites in $P A X 3$ gene in fetal neural tissues were positively correlated with PAH concentrations in maternal serum. In line with these findings in human subjects, our mouse experiment showed that the methylation level of Pax3 was elevated after $\mathrm{BaP}$ treatment, indicating that the presence of $\mathrm{BaP}$ impacts $P a x 3$ methylation regulation. These findings support the hypothesis that hypermethylation of $\operatorname{Pax} 3$
TAC

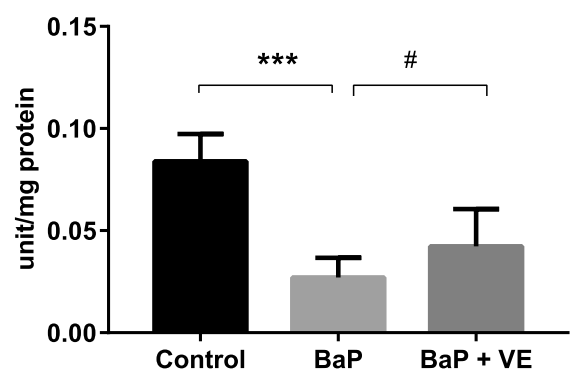

SOD

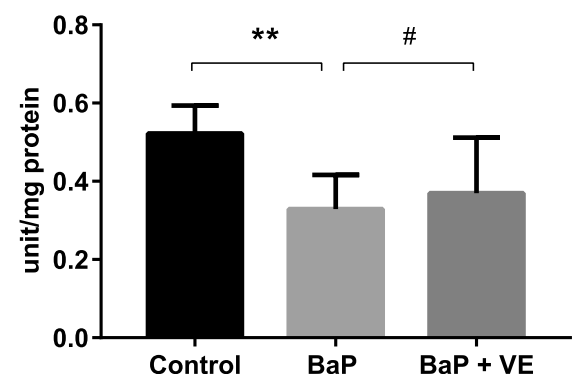

Fig. 4 The effects of BaP and vitamin E on TAC and SOD activity in E10.5 mouse embryos. Data were expressed as mean \pm SD $(n=8-10)$. ${ }^{P} P<0.05$, ${ }^{* *} P<0.01$, ${ }^{* * *} P<0.001$, compared with control group; ${ }^{\#} P<0.05,{ }^{\# \#} P<0.01$, ${ }^{\# \#} P<0.001$ compared with vitamin E supplemented group. VE vitamin $E$ 


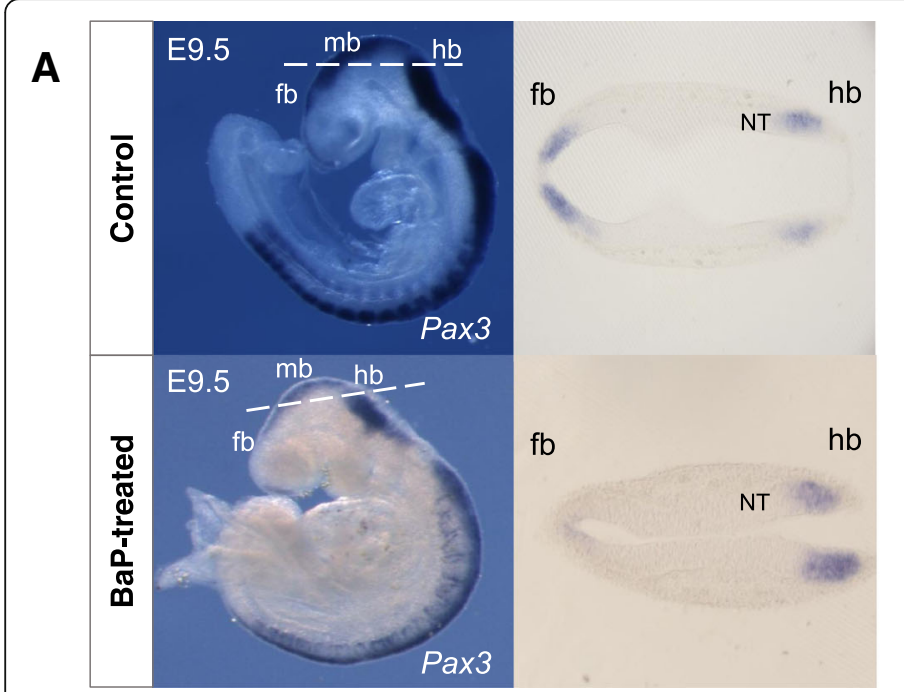

B

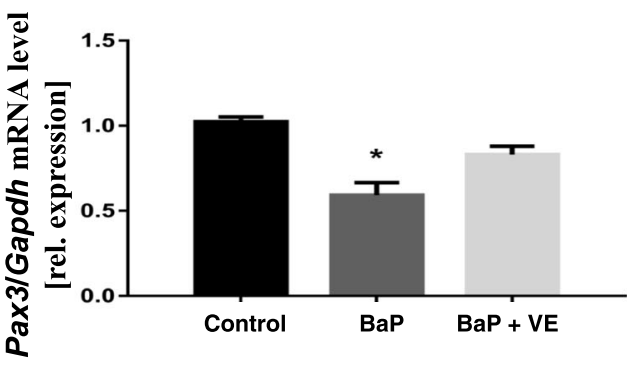

Fig. 5 The Effects of BaP and vitamin E on Pax3 gene expression in mouse embryos. a Whole mount in situ hybridization of Pax3 in E9.5 embryos. In control embryos, Pax3 mRNA was detected in the dorsal neural tube and somites as expected, while less gene expression was seen in the forebrain and midbrain of embryos exposed to BaP. The dashed lines on the whole mount panels indicate the orientation of respective sections. b Relative quantitative real-time PCR of Pax3 mRNA from E10.5 embryos exposed to BaP and co-supplemented with vitamin E. Values were normalized against Gapdh and represented as mean \pm SE $(n=5-8)$. ${ }^{*} p<0.05$, compared with control group. fb forebrain, $m b$ midbrain, $h b$ hindbrain, NT neuroepithelium, VE vitamin E

gene is involved in abnormal closure of neural tube secondary to PAH exposure.

In considering how BaP exposure could affect methylation, accumulating evidence has demonstrated that oxidative stress in response to various environmental insults or maternal dietary factors is responsible for aberrant DNA methylation [40-43]. In our study, hypermethylation of Pax3 and repressed gene expression, along with reduced TAC level, were observed in BaP-treated mouse embryos. Co-administration with vitamin $\mathrm{E}$ could rescue NTDs induced by BaP, partly normalized the TAC level, and attenuate the hypermethylation of $\operatorname{Pax} 3$ and the repressed gene expression. All these findings support the postulation that the oxidative stress may be causally involved in aberrant DNA methylation in the BaP-treated group, which might be the underlying mechanism for the development of NTDs caused by BaP. Consistent with this idea, a previous investigation revealed that epigallocatechin gallate, which is the major polyphenol in green tea and is believed to act as an antioxidant, could block hypermethylation of several neural tube closure essential genes induced by maternal diabetes, including Pax3 [30]. Although it is not precisely known, previous investigations proposed that oxidative stress could influence the synthesis of S-adenosylmethionine [44], the expression of DNA methyltransferase [45], and the activity of the ten eleven translocation enzymes [46], which are all critical for DNA methylation. On the other hand, epigenetic regulation may also contribute to impairment of antioxidant gene expression [47]. In this regard, the ROS production and epigenetic regulation establish an interconnected cycle, which would amplify external factors toward the progression of pathological disorders.

DNA methylation is considered tissue-specific and therefore appropriate biological samples for methylation study are crucial. One advantage of our study is that neural tissues from fetuses were used for assessment of methylation, which makes our methylation study more relevant to the outcome, as compared to those that used blood DNA as the surrogate [15]. The limitation is that we did not investigate the expression of $P A X 3$ gene in our human subjects, as fresh neural tissue samples of terminated NTD cases for RNA assay are extremely difficult to collect. Although our mouse experiment may provide a clue, additional studies are needed to correlate the methylation levels of the $P A X 3$ gene body with $P A X 3$ gene or protein expression values. Another limitation is that more case mothers reported folic acid supplementation than control mothers, which might cause the differences in methylation levels between the two groups. However, when analyses were performed by folic acid supplementation status in the case group, no differences in the methylation level of $P A X 3$ were found between those with or those without folic acid use (Additional file 4: Figure S1), suggesting that folic acid has no impact on methylation level in the present study.

\section{Conclusions}

In conclusion, we found that hypermethylation of $\mathrm{Pax} 3$ and the downregulation of the gene may be important 
events in the development of NTDs during embryogenesis. A relationship exists between higher maternal serum PAH concentrations and PAX3 hypermethylation in fetal neural tissues, which is further supported by our mouse experiments. In addition, oxidative stress may be involved in the process of environmental exposure and methylation modification. Our study provides novel evidence on the interaction between genetic and environmental factors in the etiology of NTDs.

\section{Methods}

\section{Study subjects}

The human subjects were recruited from rural counties in Shanxi province of northern China (Pinding, Zezhou, Xiyang, Shouyang, and Taigu), where NTD prevalence is among the highest in the world [48]. As described in more detail in our previous report [48], NTD cases were terminated fetuses affected by an NTD; controls were terminated non-malformed fetuses. Information on maternal sociodemographic characteristics, lifestyle, and folic acid supplementation was collected through in-person interviews and by viewing medical records. Maternal venous blood samples were collected at pregnancy termination. Fetal spinal cord and brain tissues were collected by autopsy performed by experienced pathologists. All samples were stored at $-80{ }^{\circ} \mathrm{C}$ until assay. The study protocol was approved by the Institutional Review Board of Peking University, and written informed consent was obtained from all participating women.

\section{Experimental animals}

ICR mice of 8-9 weeks old were used, as described previously [22]. Briefly, BaP (Sigma, USA) dissolved in corn oil was intraperitoneally administered into pregnant mice from E7 for four consecutive days (250-350 mg/ $\mathrm{kg}$ ). Mice in the vitamin $\mathrm{E}$ co-exposure group were fed with chow supplemented with the water-soluble $( \pm)$ - $\alpha$-tocopherol succinate form of vitamin E (Sigma, USA) beginning from E0.5 $(0.125 \%, w / w)$ and treated with BaP from E7 $(250 \mathrm{mg} / \mathrm{kg})$. On E10.5, embryos were collected and carefully inspected for NTDs. All experimental procedures were approved by the Institutional Animal Care and Use Committee of Peking University (certificate no. LA2013-36).

\section{DNA methylation level analysis}

DNA from neural tissues of human subjects was extracted with QIAamp DNA Mini Kit (QIAGEN, Hilden, Germany) and E10.5 mouse DNA was extracted from frozen neural tissues using TIANamp Genomic DNA Kit (TIANGEN Biotech, Beijing, China). Then, 500 ng of genomic DNA from each sample was bisulfite-treated with EZ DNA methylation kit (Zymo Research, CA,
USA). The bisulfite conversion reaction was performed in duplicate for each sample to minimize potential bias caused by variable conversion efficiency, and pooled bisulfite-treated DNA was used for subsequent analysis. Human methylation study was performed in two phases. In phase 1, Infinium HumanMethylation450 BeadChip assay (450K, Illumina, San Diego, CA, USA) was used for genomic methylation assay of bisulfite-treated DNA from ten NTD cases and eight non-malformed controls, which has been described in detail in our previous study [23]. Methylation data of $P A X 3$ gene was extracted from the array data and used for validation in the next phase.

In phase 2, 73 NTD cases and 29 non-malformed control fetuses were included. The locations of the amplicons to target the aberrant $\mathrm{CpG}$ regions are shown in Fig. 1a. Bisulphite DNA was amplified by PCR and primers for the $P A X 3$ gene were designed using the online tool Epidesigner (www.epidesigner.com). Primer sequences are listed in Additional file 5: Table S4. After reverse transcription, fragmentation, and analysis on a mass spectrometer (Sequenom, Inc., San Diego, USA), EpiTYPER Analyzer software was used for translating mass signal patterns into quantitative DNA methylation levels of different $\mathrm{CpG}$ sites.

Methylation analysis of mouse neural tissues was assayed with the same methods performed in phase 2 of the human methylation study. The regions of gene sequence analyzed were the same as those in human PAX3 gene and three amplicons were designed, with two in the promoter region and one in the body region, as shown in Fig. 1b. See Additional file 6: Table S5 for PCR primer sequences.

\section{PAHs analysis}

PAH concentrations in maternal blood were determined with an Agilent 7890A-5975C gas chromatograph and mass spectrometer equipped with a HP-5MS capillary column $(30 \mathrm{~m} \times 0.25 \mathrm{~mm} \times 0.25 \mu \mathrm{m})$, as described previously [24]. In the present study, 51 mother-fetus pairs were available for PAH-methylation correlation analysis.

\section{Oxidative stress assessment}

The antioxidant capacity was determined by the reduced ferric reducing antioxidant power (FRAP) assay according to the manufacturer's instructions (Beyotime Institute of Biotechnology, China) for determining the level of TAC of mouse embryos. The TAC aims to measure both small molecule and protein antioxidants, including polyphenols, flavonoids, vitamins, and enzymes like glutathione peroxidase and superoxide dismutase. Briefly, neural tissues from E10.5 embryo samples were homogenized in PBS. Freshly prepared FRAP reagent was warmed to $37{ }^{\circ} \mathrm{C}$ before use. Then, $5 \mu \mathrm{l}$ of the diluted sample was added to $180 \mu \mathrm{l}$ of the FRAP reagent. 
The absorbance of the mixture was measured at $593 \mathrm{~nm}$ using a Synergy 2 Multi-Mode Microplate Reader (BioTek, USA) after incubation for $4 \mathrm{~min}$. The TAC of each sample was calculated from the standard curve constructed using $\mathrm{FeSO}_{4}$ solution, and the results were expressed as $\mathrm{mmol} / \mathrm{g}$ protein, adjusted according to the protein concentration of the samples. All samples were loaded in duplicate. Protein concentration was determined using the BCA protein assay kit (Beyotime Institute of Biotechnology, China).

SOD activity was determined using the total superoxide dismutase assay kit with WST-8 (Beyotime Institute of Biotechnology, China). Briefly, $20 \mu$ l of sample (E10.5 embryo samples were homogenized in lysis buffer) was mixed with $160 \mu \mathrm{l}$ of WST-8/enzyme working solution. Then, $20 \mu \mathrm{l}$ of reaction triggering working solution was added. After incubation at $37{ }^{\circ} \mathrm{C}$ for $30 \mathrm{~min}$, the absorbance was determined at $450 \mathrm{~nm}$ using a Synergy 2 Multi-Mode Microplate Reader (BioTek, USA). The SOD activity was expressed as U/mg protein.

\section{RNA isolation and real-time PCR}

RNA was isolated from E10.5 embryos neural tissues using Trizol (Invitrogen); genomic DNA was removed by DNase I digestion (DNA-free, Ambion) and then reverse-transcribed using random hexamers (Superscript VILO cDNA synthesis kit). The abundance of mRNA of Pax3 were analyzed using real-time PCR (iTaq ${ }^{\mathrm{Tm}}$ Universal SYBR Green Supermix, BioRad) on a 7500 Fast Real Time PCR system (Applied Biosystems), with each sample analyzed in triplicate. Primers are listed in Additional file 7: Table S6. Relative quantification of gene expression level was normalized according to the Gapdh gene expression.

\section{Whole mount in situ hybridization}

Whole mount in situ hybridization on E9.5 control embryos and embryos treated with $\mathrm{BaP}$ was performed according to the procedures described by Yun et al. [49]. Pax3 probe was cloned by real-time PCR into pGEM-T (Promega) and used to generate digoxygenin-labeled cRNA probes by reverse transcription using T7 RNA polymerase (Roche). For detection, anti-digoxigenin-AP antibody (1:2000, Roche) in 1\% sheep serum was used and incubated overnight. Color detection was carried out using NBT/BCIP developing solution (Roche) in NTMT. After color development, embryos were imaged with a DFC490 camera (Leica), and then embedded. Sections of $40 \mu \mathrm{m}$ thickness were obtained using a vibratome.

\section{Statistical analyses}

In the human subject study, differences in proportions of population characteristics between NTD cases and controls were examined with Pearson's $x^{2}$ test. Independent $t$ test was performed to evaluate the difference in methylation of CpGs between NTDs and controls in phase 1, and adjusted for multiple testing with the Benjamini-Hochberg false discovery rate (FDR) methods. In phase 2, Shapiro-Wilk test was used to examine the distribution of methylation values of NTD cases and controls, and independent samples $t$ test was used to identify $\mathrm{CpG}$ sites that were differentially methylated between cases and controls. Odds ratio (OR) was calculated by logistic regression to evaluate the association between higher methylation levels of $P A X 3$ with the risk of NTDs, adjusting for maternal education, occupation, periconceptional folic acid use, fetal sex, and gestational age. Correlation between differentially methylated CpG sites in neural tissues and $\mathrm{PAH}$ concentrations in maternal serum was analyzed with Spearman's correlation analysis. In the mouse study, data on methylation level of Pax3 gene, oxidative stress markers, and the abundance of mRNA were presented as mean $\pm \mathrm{SE}(\mathrm{SD})$. A one-way analysis of variance (ANOVA) followed by LSD (equal variances assumed) or Dunnett's T3 (equal variances not assumed) was used for testing the differences between groups. A two-tail $P$ value of $<0.05$ was considered statistically significant. Statistical analyses were conducted using SPSS 18.0.

\section{Additional files}

Additional file 1: Table S1. Characteristics of NTD cases and controls in phase 1 for methylation assay. (DOCX $18 \mathrm{~kb}$ )

Additional file 2: Table S2. Methylation of PAX3 gene in phase 1 using the HumanMethylation450 BeadChip assay. (DOCX $24 \mathrm{~kb}$ )

Additional file 3: Table S3. Correlation analysis of DNA methylation of PAX3 in fetal neural tissues and PAH concentrations in maternal serum ( $N$ = 51 mother-fetus pairs). (DOCX $22 \mathrm{~kb}$ )

Additional file 4: Figure S1. PAX3 methylation pattern assayed by Sequenom EpiTYPER in NTD cases with and without folic acid supplementation. (DOCX $148 \mathrm{~kb}$ )

Additional file 5: Table S4. The PCR primer sequences in Sequenom EpiTYPER sequencing. (DOCX $15 \mathrm{~kb}$ )

Additional file 6: Table S5. The PCR primer sequences for mouse methylation analysis. (DOCX $15 \mathrm{~kb}$ )

Additional file 7: Table S6. Sequences of primers for real-time PCR in mouse study. (DOCX $15 \mathrm{~kb}$ )

\section{Abbreviations}

BaP: Benzo[a]pyrene; FDR: False discovery rate; FRAP: Ferric reducing antioxidant power; HM450K: HumanMethylation450 BeadChip; NTDs: Neural tube defects; PAH: Polycyclic aromatic hydrocarbon; ROS: Reactive oxygen species; SOD: Superoxide dismutase; TAC: Total antioxidant capacity acknowledged. All participants contributing to the original study subjects recruit are acknowledged. 


\section{Funding}

This work was supported by the National Key Research and Development Program, Ministry of Science and Technology, the Peoples' Republic of China (Grant No. 2016YFC1000501), and grants from the National Natural Science Foundation of China (Grant No. 31371523 and 81773441), the Ministry of Education of China (Grant No. 20130001110064), and the UK Medical Research Council (N003713, K022741). NG is supported by Great Ormond Street Hospital Children's Charity and by the National Institute for Health Research Biomedical Research Centre at Great Ormond Street Hospital for Children NHS Foundation Trust and University College London.

\section{Availability of data and materials}

The data sets supporting the results of this article are included within the article and its additional files.

\section{Authors' contributions}

AR and LW conceived and designed the study. SL performed the experiments, conducted data analyses, interpreted the data, and drafted the manuscript. YH and CS helped with the experiments. AR, LW, CS, and NG critically revised the paper. NG helped provide reagents, materials, and experimental infrastructure. $\mathrm{L}$ and $\mathrm{ZL}$ contributed to human subject recruitment. All authors read and approved the definitive version of the manuscript.

\section{Ethics approval and consent to participate}

The study protocol was approved by the Institutional Review Board of Peking University, and written informed consent was obtained from all participating women. The animal experiments were approved by the Institutional Animal Care and Use Committee of Peking University (certificate no. LA2013-36).

\section{Consent for publication}

Not applicable.

\section{Competing interests}

The authors declare that they have no competing interests.

\section{Publisher's Note}

Springer Nature remains neutral with regard to jurisdictional claims in published maps and institutional affiliations.

\section{Author details}

${ }^{1}$ Institute of Reproductive and Child Health, National Health Commission Key Laboratory of Reproductive Health, and Department of Epidemiology and Biostatistics, School of Public Health, Peking University Health Centre, Peking University, Beijing 100191, China. 'Division of Birth Cohort Study, and Department of Neonatal Surgery, Guangzhou Women and Children's Medical Centre, Guangzhou Medical University, Guangzhou, China. ${ }^{3}$ Developmental Biology and Cancer Programme, UCL Great Ormond Street Institute of Child Health, University College London, London WC1N 1EH, UK.

\section{Received: 21 August 2018 Accepted: 8 January 2019}

\section{Published online: 21 January 2019}

\section{References}

1. Mitchell LE. Epidemiology of neural tube defects. Am J Med Genet C Semin Med Genet. 2005;135c(1):88-94

2. Greene ND, Stanier P, Copp AJ. Genetics of human neural tube defects. Hum Mol Genet. 2009:18(R2):R113-29.

3. Harris MJ, Juriloff DM. Mouse mutants with neural tube closure defects and their role in understanding human neural tube defects. Birth Defects Res A Clin Mol Teratol. 2007;79(3):187-210

4. Wilde JJ, Petersen JR, Niswander L. Genetic, epigenetic, and environmental contributions to neural tube closure. Annu Rev Genet. 2014:48:583-611.

5. Greene ND, Stanier P, Moore GE. The emerging role of epigenetic mechanisms in the etiology of neural tube defects. Epigenetics. 2011;6(7): 875-83

6. Weinhold B. Epigenetics: the science of change. Environ Health Perspect. 2006;114(3):A160-7.

7. Morgan HD, Santos F, Green K, Dean W, Reik W. Epigenetic reprogramming in mammals. Hum Mol Genet. 2005;14 Spec No 1:R47-58.
8. Kim JK, Samaranayake M, Pradhan S. Epigenetic mechanisms in mammals. Cell Mol Life Sci. 2009;66(4):596-612.

9. Wang L, Wang F, Guan J, Le J, Wu L, Zou J, et al. Relation between hypomethylation of long interspersed nucleotide elements and risk of neural tube defects. Am J Clin Nutr. 2010;91(5):1359-67.

10. Wu L, Wang L, Shangguan S, Chang S, Wang Z, Lu X, et al. Altered methylation of IGF2 DMRO is associated with neural tube defects. Mol Cell Biochem. 2013;380(1-2):33-42.

11. Bai B, Zhang Q, Liu X, Miao C, Shangguan S, Bao Y, et al. Different epigenetic alterations are associated with abnormal IGF2/lgf2 upregulation in neural tube defects. PLoS One. 2014;9(11):e113308.

12. Liu Z, Wang Z, Li Y, Ouyang S, Chang H, Zhang T, et al. Association of genomic instability, and the methylation status of imprinted genes and mismatch-repair genes, with neural tube defects. Eur J Hum Genet. 2012;20(5):516-20.

13. Shangguan $S$, Wang $L$, Chang $S$, Lu X, Wang Z, Wu L, et al. DNA methylation aberrations rather than polymorphisms of FZD3 gene increase the risk of spina bifida in a high-risk region for neural tube defects. Birth Defects Res A Clin Mol Teratol. 2015;103(1):37-44.

14. Tian $T$, Wang $L$, Shen $Y$, Zhang B, Finnell RH, Ren A. Hypomethylation of GRHL3 gene is associated with the occurrence of neural tube defects. Epigenomics. 2018;10(7):891-901.

15. Rochtus A, Izzi B, Vangeel E, Louwette $S$, Wittevrongel C, Lambrechts D, et al. DNA methylation analysis of Homeobox genes implicates HOXB7 hypomethylation as risk factor for neural tube defects. Epigenetics. 2015; 10(1):92-101.

16. Phelan SA, Ito M, Loeken MR. Neural tube defects in embryos of diabetic mice: role of the Pax-3 gene and apoptosis. Diabetes. 1997:46(7):1189-97.

17. Greene ND, Massa V, Copp AJ. Understanding the causes and prevention of neural tube defects: insights from the splotch mouse model. Birth Defects Res A Clin Mol Teratol. 2009:85(4):322-30.

18. Pingault V, Ente D, Dastot-Le Moal F, Goossens M, Marlin S, Bondurand N. Review and update of mutations causing Waardenburg syndrome. Hum Mutat. 2010;31(4):391-406.

19. Hol FA, Hamel BC, Geurds MP, Mullaart RA, Barr FG, Macina RA et al. A frameshift mutation in the gene for PAX3 in a girl with spina bifida and mild signs of Waardenburg syndrome. J Med Genet. 1995:32(1):52-6.

20. Lu W, Zhu H, Wen S, Laurent C, Shaw GM, Lammer EJ, et al. Screening for novel PAX3 polymorphisms and risks of spina bifida. Birth Defects Res A Clin Mol Teratol. 2007;79(1):45-9.

21. Ren A, Qiu X, Jin L, Ma J, Li Z, Zhang L, et al. Association of selected persistent organic pollutants in the placenta with the risk of neural tube defects. Proc Natl Acad Sci U S A. 2011;108(31):12770-5.

22. Lin S, Ren A, Wang L, Huang Y, Wang Y, Wang $C$, et al. Oxidative stress and apoptosis in benzo[a]pyrene-induced neural tube defects. Free Radic Biol Med. 2018:116:149-58.

23. Wang L, Lin S, Zhang J, Tian T, Jin L, Ren A. Fetal DNA hypermethylation in tight junction pathway is associated with neural tube defects: a genomewide DNA methylation analysis. Epigenetics. 2017;12(2):157-65.

24. Wang B, Jin L, Ren A, Yuan Y, Liu J, Li Z, et al. Levels of polycyclic aromatic hydrocarbons in maternal serum and risk of neural tube defects in offspring Environ Sci Technol. 2015:49(1):588-96.

25. Fleming A, Copp AJ. Embryonic folate metabolism and mouse neural tube defects. Science. 1998:280(5372):2107-9.

26. Fleming A, Copp AJ. A genetic risk factor for mouse neural tube defects: defining the embryonic basis. Hum Mol Genet 2000:9(4):575-581.

27. Nye JS, Balkin N, Lucas H, Knepper PA, McLone DG, Charrow J. Myelomeningocele and Waardenburg syndrome (type 3 ) in patients with interstitial deletions of 2q35 and the PAX3 gene: possible digenic inheritance of a neural tube defect. Am J Med Genet. 1998;75(4):401-8.

28. Agopian AJ, Bhalla AD, Boerwinkle E, Finnell RH, Grove ML, Hixson JE, et al. Exon sequencing of PAX3 and T (brachyury) in cases with spina bifida. Birth Defects Res A Clin Mol Teratol. 2013:97(9):597-601.

29. Wei D, Loeken MR. Increased DNA methyltransferase 3b (Dnmt3b)-mediated $\mathrm{CpG}$ island methylation stimulated by oxidative stress inhibits expression of a gene required for neural tube and neural crest development in diabetic pregnancy. Diabetes. 2014;63(10):3512-22.

30. Zhong J, Xu C, Reece EA, Yang P. The green tea polyphenol EGCG alleviates maternal diabetes-induced neural tube defects by inhibiting DNA hypermethylation. Am J Obstet Gynecol. 2016;215(3):368. e1-.e10

31. Rochtus A, Winand $\mathrm{R}$, Laenen $\mathrm{G}$, Vangeel $\mathrm{E}$, Izzi B, Wittevrongel $\mathrm{C}$, et al. Methylome analysis for spina bifida shows SOX18 hypomethylation as a risk 
factor with evidence for a complex (epi)genetic interplay to affect neural tube development. Clin Epigenetics. 2016;8:108.

32. Bell AC, Felsenfeld G. Methylation of a CTCF-dependent boundary controls imprinted expression of the lgf2 gene. Nature. 2000;405(6785):482-5.

33. Zhang X, Yazaki J, Sundaresan A, Cokus S, Chan SW, Chen H, et al. Genomewide high-resolution mapping and functional analysis of DNA methylation in arabidopsis. Cell. 2006;126(6):1189-201.

34. Lev Maor G, Yearim A, Ast G. The alternative role of DNA methylation in splicing regulation. Trends Genet. 2015;31(5):274-80.

35. Yearim A, Gelfman S, Shayevitch R, Melcer S, Glaich O, Mallm JP, et al. HP1 is involved in regulating the global impact of DNA methylation on alternative splicing. Cell Rep. 2015;10(7):1122-34.

36. Tang WY, Levin L, Talaska G, Cheung YY, Herbstman J, Tang D, et al. Maternal exposure to polycyclic aromatic hydrocarbons and 5 '-CpG methylation of interferon-gamma in cord white blood cells. Environ Health Perspect. 2012;120(8):1195-200

37. Herbstman JB, Tang D, Zhu D, Qu L, Sjodin A, Li Z, et al. Prenatal exposure to polycyclic aromatic hydrocarbons, benzo[a]pyrene-DNA adducts, and genomic DNA methylation in cord blood. Environ Health Perspect. 2012; 120(5):733-8.

38. Fang $X$, Dong W, Thornton C, Willett KL. Benzo[a]pyrene effects on glycine $\mathrm{N}$-methyltransferase mRNA expression and enzyme activity in Fundulus heteroclitus embryos. Aquat Toxicol. 2010;98(2):130-8.

39. Sadikovic B, Rodenhiser DI. Benzopyrene exposure disrupts DNA methylation and growth dynamics in breast cancer cells. Toxicol Appl Pharmacol. 2006;216(3):458-68

40. Dolinoy DC, Huang D, Jirtle RL. Maternal nutrient supplementation counteracts bisphenol A-induced DNA hypomethylation in early development. Proc Natl Acad Sci U S A. 2007;104(32):13056-61.

41. Bernal AJ, Dolinoy DC, Huang D, Skaar DA, Weinhouse C, Jirtle RL. Adaptive radiation-induced epigenetic alterations mitigated by antioxidants. FASEB $J$. 2013;27(2):665-71.

42. Franco R, Schoneveld O, Georgakilas AG, Panayiotidis MI. Oxidative stress, DNA methylation and carcinogenesis. Cancer Lett. 2008;266(1):6-11.

43. Angelini F, Pagano F, Bordin A, Milan M, Chimenti I. The impact of environmental factors in influencing epigenetics related to oxidative states in the cardiovascular system. Oxid Med Cell Longev. 2017:2017:2712751.

44. Hitchler MJ, Domann FE. An epigenetic perspective on the free radical theory of development. Free Radic Biol Med. 2007;43(7):1023-36.

45. Weitzman SA, Turk PW, Milkowski DH, Kozlowski K. Free radical adducts induce alterations in DNA cytosine methylation. Proc Natl Acad Sci U S A. 1994;91(4):1261-4.

46. Chia N, Wang L, Lu X, Senut MC, Brenner C, Ruden DM. Hypothesis: environmental regulation of 5-hydroxymethylcytosine by oxidative stress. Epigenetics. 2011;6(7):853-6.

47. Cyr AR, Hitchler MJ, Domann FE. Regulation of SOD2 in cancer by histone modifications and $\mathrm{CpG}$ methylation: closing the loop between redox biology and epigenetics. Antioxid Redox Signal. 2013;18(15):1946-55.

48. Liu J, Zhang L, Li Z, Jin L, Zhang Y, Ye R, et al. Prevalence and trend of neural tube defects in five counties in Shanxi province of northern China, 2000 to 2014. Birth Defects Res A Clin Mol Teratol. 2016;106(4):267-74.

49. Pai YJ, Leung KY, Savery D, Hutchin T, Prunty H, Heales S, et al. Glycine decarboxylase deficiency causes neural tube defects and features of nonketotic hyperglycinemia in mice. Nat Commun. 2015;6:6388

\section{Ready to submit your research? Choose BMC and benefit from:}

- fast, convenient online submission

- thorough peer review by experienced researchers in your field

- rapid publication on acceptance

- support for research data, including large and complex data types

- gold Open Access which fosters wider collaboration and increased citations

- maximum visibility for your research: over $100 \mathrm{M}$ website views per year

At BMC, research is always in progress.

Learn more biomedcentral.com/submissions 